\title{
Resurgence of Middle East Respiratory Syndrome Coronavirus Outbreak in Saudi Arabia
}

\author{
Masoud Mardani ${ }^{1, *}$ \\ ${ }^{1}$ Infectious Diseases and Tropical Medicine Research Center, Shahid Beheshti University of Medical Sciences, Tehran, IR Iran \\ ${ }^{*}$ Corresponding author: Masoud Mardani, Infectious Diseases and Tropical Medicine Research Center, Shahid Beheshti University of Medical Sciences, Tehran, IR Iran. Tel: \\ +98-21224399638, Fax: +98-2122439964, E-mail: drmasoudmardani@yahoo.com \\ Received: July 12, 2015; Accepted: July 20, 2015
}

Keywords: Middle East Respiratory Syndrome (MERS); Outbreak; South Korea

Middle East Respiratory Syndrome (MERS) is a respiratory disease caused by a newly recognized coronavirus (MERS-Co). It was first reported in 2012 in Saudi Arabia and since then linked to countries in or near the Arabian Peninsula (United Arab Emirates, Qatar, Oman, Jordan, Kuwait, Yemen, and Lebanon) and Iran. As of May 2014, two cases had been reported in the United States, both in men who had recently returned from Saudi Arabia (1). Also Iran has been reported plenty of cases but fortunately the outbreak subsided (2).

Recently South Korea had a large outbreak of the MERS and was blamed on locating every person exposed to the disease. Many of the 180 MERS cases in South Korea, which make up the largest outbreak of the disease outside Saudi Arabia, were caught from sufferers encountered in emergency wards before they were diagnosed, during a wait for hospital places; unfortunately 30 out of 180 infected patients expired (3).

MERS is typically characterized by cough, fever, and shortness of breath. Most individuals with confirmed MERS develop acute respiratory illness and among the 536 cases reported since May 12, 2014, the mortality rate was $30 \%$. However, the number of cases and fatalities reported in Saudi Arabia has recently been revised following alleged underreporting. The updated total number of cases in that country has been increased to 688 from the previous count of 575 and the total number of deaths has been increased to 282 from 190, representing a mortality rate of more than $40 \%$ (4).

MERS-CoV causes a spectrum of diseases from asymptomatic infection to mild illness and to finally life threatening infection leading to death. Common symptoms are acute respiratory illness with fever, cough, shortness of breath, and breathing difficulties. Most patients have had pneumonia. Many have also had gastrointestinal symptoms like diarrhea. Some patients have had kidney failure. In people with immune deficiencies, the disease may have an atypical presentation. The majority of patients have had at least one comorbid condition, such as diabetes, heart disease, hypertension, or pulmonary disease (5).

Laboratory testing for MERS-CoV is not routinely available, but a real-time reverse transcription polymerase chain reaction (RT-PCR) assay for MERS-CoV is available in reference laboratory health departments. Specimens for rRT-PCR testing include specimens from the upper and lower respiratory tract (for example, nasopharyngeal swab, sputum, and bronchoalveolar lavage), blood and stool. Within 14 days of symptom onset, multiple specimens should be obtained from different sites and at different time intervals for rRT-PCR testing. MERS-CoV viral load and genome yield were higher in specimens of lower respiratory tract origin. Serology is included if the time elapsed was more than 14 day from onset. Interpretation of MERS-CoV laboratory results is provided with case definition (6).

Currently, no vaccine exists for MERS, and no specific treatment has been recommended. At present, management is supportive. However, at least one group has recommended consideration of interferon alfa-2b plus ribavirin in the management of MERS-CoV cases because the combination's efficacy was seen in rhesus macaques with $\operatorname{MERS}(7)$.

Travelers who have recently visited countries in or near the Arabian Peninsula are advised to contact their healthcare provider if they develop MERS symptoms (i.e. fever, cough, shortness of breath) within 14 day of travel (8). Individuals who have been in recent contact with a symptomatic person newly traveled to the Arabian Peninsula should also be evaluated for MERS-CoV infection (8).

Because of an ongoing 2015 outbreak of MERS in South Korean healthcare facilities, Centers for Disease Control and Prevention (CDC) has advised clinicians to ask patients with serious respiratory problems whether they have recently been in a healthcare facility in South Korea

Copyright (C) 2015, Infectious Diseases and Tropical Medicine Research Center. This is an open-access article distributed under the terms of the Creative Commons Attribution-NonCommercial 4.0 International License (http://creativecommons.org/licenses/by-nc/4.0/) which permits copy and redistribute the material just in noncommercial usages, provided the original work is properly cited. 
to screen for MERS-CoV. A patient presenting with fever and pneumonia or acute respiratory distress syndrome should be evaluated for MERS if he or she had been a patient, worker, or visitor in a South Korean healthcare facility within 14 day of symptom onset (2).

In patients who meet these screening criteria, $\mathrm{CDC}$ recommends evaluation for common causes of community-acquired pneumonia based on clinical presentation, epidemiologic, and surveillance information. Testing for MERS-CoV and other respiratory pathogens can be performed concurrently. Results that confirm a different respiratory pathogen should not necessarily preclude testing for MERS-CoV (2).

Standard contact and airborne precautions are recommended for the management of hospitalized patients with known or suspected MERS-CoV infection. The complete CDC guidance can be found on the CDC and WHO websites. In addition, personal protective equipment for healthcare personnel, including gloves, gowns, eye protection (goggles or face shield), and respiratory protection (at least as protective as fit-tested NIOSH-certified disposable $\mathrm{N}-95$ filtering facepiece respirator) must be utilized (2).

To sum up, it seems that geographical district of MERS$\mathrm{CoV}$ has been expanded, which can be a serious threat for public health. Hence adequate knowledge of physicians and medical staff is the best way to deal with this contagious disease.

\section{Acknowledgements}

The author appreciates Bita Pourkaveh for her contribution in collecting data.

\section{References}

1. Rha B, Rudd J, Feikin D, Watson J, Curns AT, Swerdlow DL, et al. Update on the epidemiology of Middle East respiratory syndrome coronavirus (MERS-CoV) infection, and guidance for the public, clinicians, and public health authorities - January 2015. MMWR Morb Mortal Wkly Rep. 2015;64(3):61-2.

2. WHO.. News and Top Stories. 2015. Available from: www.who.int

3. Cowling BJ, Park M, Fang VJ, Wu P, Leung GM, Wu JT. Preliminary epidemiological assessment of MERS-CoV outbreak in South Korea, May to June 2015. Euro Surveill. 2015;20(25).

4. Khalafalla AI, Lu X, Al-Mubarak AI, Dalab AH, Al-Busadah KA Erdman DD. MERS-CoV in Upper Respiratory Tract and Lungs of Dromedary Camels, Saudi Arabia, 2013-2014. Emerg Infect Dis. 2015;21(7):1153-8.

5. Mardani M, Abtahian Z, Mardani K. Overview of the Middle East Respiratory Syndrome (MERS) coronavirus. Pajoohandeh J. 2014;19(1):1-12.

6. Mardani M. Middle East Respiratory Syndrome Outbreak Threat Becomes More Urgent. Arch Clin Infect Dis. 2014;9(2):e20693.

7. Falzarano D, de Wit E, Rasmussen AL, Feldmann F, Okumura A Scott DP, et al. Treatment with interferon-alpha2b and ribavirin improves outcome in MERS-CoV-infected rhesus macaques. Nat Med.2013;19(10):1313-7.

8. de Groot RJ, Baker SC, Baric RS, Brown CS, Drosten C, Enjuanes L, et al. Middle East respiratory syndrome coronavirus (MERS$\mathrm{CoV})$ : announcement of the Coronavirus Study Group. J Virol. 2013;87(14):7790-2. 